\title{
CUSTOMIZAÇÃO EM MASSA DE ITENS PARA AUTOMAÇÃO RESIDENCIAL EM EDIFÍCIOS HABITACIONAIS
}

\author{
Débora Nascimento (debora.nascimento@ifmg.edu.br) - Universidade Federal de Santa Catarina \\ Diego Castro Fettermann (d.fettermann@ufsc.br) - Universidade Federal de Santa Catarina
}

\section{RESUMO}

A estratégia de Customização em Massa já é adotada em diversas empresas e indústrias, incluindo a indústria da construção civil. As necessidades dos clientes são atendidas por meio da customização de itens físicos e estruturais nas residências. Com o desenvolvimento de soluções IoT e evolução das tecnologias de comunicação e informação, se verifica um grande potencial de atendimento de necessidades específicas dos clientes por meio da incorporação de itens de automação nas residências. Assim, o objetivo deste artigo é identificar possíveis itens de automação residencial para customização em massa de empreendimentos habitacionais. Por meio da análise da literatura foi possível construir um quadro que apresenta categorias e itens que caracterizam uma smart-home, sendo estes itens potenciais variáveis de automação residencial para customização em massa de edifícios habitacionais, como a categoria conforto que engloba itens de controle de temperatura, iluminação e ventilação.

Palavras chave: automação residencial; customização em massa; construção; habitação; smart-home 


\section{INTRODUÇÃ̃}

O termo Customização em Massa (CM) foi utilizado, primeiramente, no final da década de 1980 como forma de atender a uma maior variedade de produtos demandada pelos clientes (DAVIS, 1987). Mais tarde, Pine II (1993) desenvolveu uma definição de CM mais abrangente que envolve o desenvolvimento, a produção e a comercialização de bens e serviços acessíveis com variedade e customização próximo ao que os clientes desejam. Posteriormente, foram registrados estudos que buscaram aperfeiçoar esta estratégia, apresentando uma maior ênfase na possibilidade de personalização individual do produto, definida por Personalização em Massa (PM) (WANG et al., 2017). Apesar do aperfeiçoamento da PM, ambas estratégias estão relacionadas à habilidade em disponibilizar produtos ou serviços customizados por meio de processos flexíveis a custos relativamente baixos. (FOGLIATTO; SILVEIRA; BORENSTEIN, 2012)

$\mathrm{Na}$ literatura, é possível identificar que a CM pode agregar valor para os clientes (KOTHA, 1995; PINE II, 1993) através da análise de casos de sucesso reportados na literatura, como na Adidas (PILLER; LINDGENS; STEINER; 2012), no varejo de bicicletas (TEICHMANN; SCHOLL-GRISSEMANN; STOKBURGER-SAUER; 2016) e na indústria da moda e de marcas de luxo (PARK; YOO; 2018; YOO; PARK; 2016). Porém, algumas empresas enfrentaram dificuldades em sua implementação, como na indústria automobilística (POLLARD; CHUO; LEE; 2008). Uma das razões desses problemas reside na dificuldade em compreender o mercado e ter disponibilidade de tecnologias adequadas para a implementação da CM. (ZIPKIN, 2001)

Na indústria da construção civil observa-se que a modularização para os projetos de prédios habitacionais tem sido o suporte para o processo de implementação da estratégia da CM. Em geral, a personalização dos edifícios residenciais ocorre nas primeiras fases de projeto (FETTERMANN; TORTORELLA; TABOADA, 2019; SCHOENWITZ et al., 2017), sendo que essa personalização são definições relacionadas às categorias como sanitárias, design interno e fachadas. (SCHOENWITZ; NAIM; POTTER, 2012)

Dentre as construções habitacionais, encontram-se pesquisas que estão buscando inserir a automação residencial como foco de seus estudos, sendo que a maioria dessas pesquisas relacionam a automação residencial com os termos smart-home ou smart-house. A automação residencial funcional é um dos valores inteligentes significativos para a caracterização de uma 
smart-home, bem como a integração das tecnologias de informação e comunicação. (GHAFFARIANHOSEINI et al., 2013)

Diversos estudos mencionam a tendência de automação residencial como um fenômeno crescente do gerenciamento das tecnologias de comunicação nas construções (BHATT; VERMA, 2015), sendo essa automação residencial vista como opção tecnológica em termos de comunicação e informação para a evolução do desenvolvimento de smart-home (GHAFFARIANHOSEINI et al., 2013) e como manifestação favorável na construção residencial por meio da computação. (LEE et al., 2014)

Na literatura, é mencionada a importância da automação residencial na vida das pessoas como uma extensão natural das tecnologias de comunicação, informação e eletrônica que desenvolverão a smart-home (CHAN et al., 2008). Dentro de poucos anos, todas as casas terão algum tipo de automação, tais como os itens de monitoramento de temperatura, iluminação, umidade do ar (SHAH; MISHRA, 2016), itens de controle de abertura e fechamento individual de janelas (KIM; LEE; LEE, 2014), itens de sistemas de segurança (BHATT; VERMA, 2015), itens de gerenciamento de alarme, temperatura, horários, iluminação (LEE et al., 2014), itens de conforto relacionados a integração de eletrodomésticos (CHAN et al., 2008; GHAFFARIANHOSEINI et al., 2013) e itens de conforto térmico. (DAUM; HALDI; MOREL, 2011)

Apesar das diversas possibilidades de customização para a implementação de automação residencial, é importante desenvolver uma compreensão clara das preferencias e necessidades dos clientes (WAN et al., 2017). Assim, este artigo busca responder: a) Quais são os itens que caracterizam uma smart-home? b) Quais são os itens demandados pelos clientes para a automação residencial em edifícios residenciais? A partir do problema de pesquisa, este trabalho apresenta como objetivo identificar, na literatura, possíveis itens de automação residencial para customização em massa de empreendimentos habitacionais. A contribuição, portanto, deste artigo é complementar a literatura com uma síntese dos itens de automação residencial apresentados pelos autores estudados e apresentar para indústria civil maior diversidade de itens customizáveis.

\section{REVISÃO TEÓRICA}

Este artigo apresenta uma análise da literatura sobre a customização em massa na indústria da construção civil para entender como funciona essa estratégia neste tipo de indústria. Definições, 
conceitos, tipos de abordagens e estudos sobre smart-home são apresentados neste artigo, baseados na literatura existente.

\subsection{Customização em massa na construção civil}

A estratégia da $\mathrm{CM}$ na construção civil tem sido apresentada, na literatura, por meio de diferentes abordagens em diferentes fases do processo. As pesquisas estão concentradas na primeira fase do processo de customização, denominada definição de opções (FETTERMANN; TORTORELLA; TABOADA, 2019). Estudos apresentam meios para reduzir a complexidade da implementação da estratégia de CM em sistemas de construção através da exploração de relações como a modularidade de produto e de processos (WANG et al., 2016). A estratégia da CM na construção de edifícios, que ainda apresentam técnicas construtivas tradicionais baseadas em concreto armado por exemplo, pode ser pouco suportada pela modularidade de produtos (FETTERMANN; TORTORELLA; TABOADA, 2019). Mas, ainda, existem vantagens na aplicação dessa estratégia, como a possibilidade de customização do produto durante o tempo de uso, o longo ciclo de vida permite vários ciclos de otimização. (FETTERMANN; TORTORELLA; TABOADA, 2019)

A complexidade da implementação da CM pode advir da complexidade da indústria de habitação, que consiste em uma mistura de pessoas, plantas, materiais, locais e novas tecnologias. Além disso, a indústria da construção tem que apresentar um rápido desenvolvimento de casas sob medida, ao mesmo tempo que impõem a regra das economias de escala. Ao ser desenvolvida a tecnologia de pré-montagem de edifícios, cria-se um facilitador da customização na construção (SCHOENWITZ; NAIM; POTTER, 2012). Assim, a ideia de buscar uma customização mais eficiente demonstrou ser apropriada para a construção habitacional, aumentando a satisfação do cliente e aumentando a participação de mercado, oferecendo casas que se aproximam das necessidades dos clientes. (SCHOENWITZ et al., 2012)

Uma habitação é um produto complexo que consiste em muitos componentes, e para satisfazer os clientes é necessário ofertar um grau de customização adequado, pois este fator pode influenciar o cliente e sua decisão de compra. Para atender a essas necessidades dos clientes de forma adequada, é necessário que as empresas construtoras habitacionais desenvolvam o alinhamento entre os seus processos e o produto customizado que é ofertado. (SCHOENWITZ et al., 2012) 
As preferências dos clientes podem ser entendidas como itens de customização na construção civil. A forma da habitação como a divisão, as funções e o tamanho dos espaços são os itens de customização em projetos de apartamento na pesquisa de Griz et al. (2017). No trabalho de Marchesi e Matt (2017) é identificado apenas um item relacionado às atividades de lazer do cliente e os demais relacionados aos espaços, áreas comuns, áreas privativas, áreas acessórias, áreas de conexão e áreas externas. Esses espaços podem ser criados, adaptados e ampliados. (TAUBE; HIROTA, 2017)

\subsection{Smart-Home}

A introdução das abordagens relacionadas a projetos inteligentes na arquitetura possibilitou o desenvolvimento do termo smart-house/smart-home, que pode ser definido como uma residência que incorporou os sensores fazendo a integração de vários sistemas, possibilitando a comunicação e promovendo o controle remoto de todos os itens (GHAFFARIANHOSEINI et al., 2013). As smart-homes possuem uma integração total com um ambiente inteligente, baseada na interrelação entre usuário e ambiente e focada na automação residencial em conjunto com a automação funcional das residências. (GHAFFARIANHOSEINI et al., 2013)

Outro conceito define uma smart-home como sendo uma residência equipada com uma rede de alta tecnologia, conectando sensores e dispositivos domésticos, aparelhos e recursos que podem ser monitorados, acessados ou controlados remotamente, e fornecer serviços que atendam às necessidades de seus habitantes (CHAN et al., 2008). Este conceito pode se referir a qualquer forma de residência, uma casa autônoma, um apartamento ou uma unidade em um conjunto habitacional social. Os sensores podem ser usados para detectar a localização de pessoas e objetos, ou coletar dados sobre estados (temperatura, uso de energia, janelas abertas); os dispositivos, aparelhos e recursos domésticos podem incluir desde máquinas de lavar roupa ou iluminação até uma interface de usuário que forneça acesso e controle de dados e serviços domésticos inteligentes (CHAN et al., 2008). O que diferencia uma smart-home de uma residência convencional é a rede que conecta e coordena todos os itens tecnológicos. (BALTAOZKAN et al., 2013)

Atentos a vários estilos de vida e por consequência a estilos de residência diferentes, integrados à comunidade local e à cidade na qual a residência está inserida, alguns pesquisadores têm desenvolvido estudos que envolvem o conceito de smart-home nessa área (GHAFFARIANHOSEINI et al., 2013). Contudo, observa-se na literatura vários estudos que 
associam o conceito de smart-homes com eficiência energética e sustentabilidade (BALTAOZKAN et al., 2013; BECKEL et al., 2014; YANG; LEE, 2014; PARK et al., 2014), visto que a smart-home oferece uma nova maneira de ver o papel que a energia desempenha na vida cotidiana. Encontram-se também estudos que visualizam a smart-home como um espaço necessário para aquelas que pessoas que necessitam de assistência especial, como idosos e portadores de alguma deficiência. (BALTA-OZKAN et al., 2013; CHAN et al., 2008)

Para atender às necessidades específicas de cada usuário da smart-homes, a estratégia da CM pode ser implementada nos empreendimentos habitacionais através de itens de automação residencial, que são identificados como itens que caracterizam o sistema integrado em uma smart-home.

No trabalho de Chan et al. (2018), alguns itens de automação residencial, como equipamentos e objetos, foram agrupados em quatro tipos de funções (suporte, monitoramento, entrega de terapêuticos e conforto) para fomentar os estudos relacionados a adequação de uma smart-home para as necessidades de idosos. A função de suporte foca nas necessidades dos usuários que possuem algum tipo de deficiência física, incluindo deficiência visual e auditiva. Os itens relacionados a esta função são equipamentos de robótica de reabilitação, robôs de companhia, cadeiras de rodas, interface especializada, geração de voz sintética para controle e comando, tela tátil, controle remoto sensível, farol audível e alarme visível. A função de monitoramento baseia-se no estilo de vida do usuário em que ele é monitorado por meio de sistemas fixos (com sensores infravermelhos), de sistemas vestíveis (usando crachá ativo e acelerômetro) e de sinais fisiológicos (com sensores externos e in vivo). A função de entregas terapêuticas agrega dispositivos que façam entregas, principalmente, de medicamentos. A função conforto agrega dispositivos domésticos inteligentes (máquina de lavar louça, máquina de lavar roupa, geladeira e fogão), objetos inteligentes (caixa de correio, armário de roupa e espelho), equipamentos de casa inteligente (sensores de presença e movimento, câmera de vídeo, interruptores magnéticos e sensores de humidade, gás e luz), equipamentos de lazer inteligente (TV e programas de cinema em casa), sistemas de comunicação interativa (comunicação com amigos e familiares em caso de emergência), equipamentos inteligentes de controle do ambiente (janelas e portas, aquecimento, iluminação, ar condicionado e ventilação) e atividade física (dispositivos fitness) (CHAN et al.; 2018).

$\mathrm{Na}$ categoria de assistente de vida os itens de automação residencial podem fornecer aos ocupantes idosos ou deficientes e seus amigos e parentes uma maior independência e 
tranquilidade, monitorando a atividade do ocupante e contatando um cuidador em caso de atividade incomum (por exemplo, não ligar a chaleira pela manhã) que sinalize um potencial acidente ou doença. Na categoria que engloba os serviços inteligentes de segurança doméstica, os itens de automação residencial podem oferecer a possibilidade de monitorar movimentos em casa e identificar potenciais intrusos, ser alertado sobre portas e janelas abertas ou programar padrões aleatórios de iluminação de salas para deter ladrões de propriedade temporariamente desocupada. A categoria de serviços de eficiência energética de smart-home auxilia os proprietários a reduzir a demanda de energia, seja diretamente (por meio de mecanismos automatizados de economia de energia, como reduzir o aquecimento em dias quentes) ou indiretamente (por exemplo, fornecendo ao usuário acesso centralizado a dados sobre sua realidade de tempo de uso de energia e conta de energia). (BALTA-OZKAN et al., 2013)

A smart-home pode apresentar estilos diferentes com características próprias a cada estilo: casa adaptativa (Universidade do Colorado), casa consciente (Instituto de Tecnologia da Georgia), "House-n Project" (MIT), "Easy Living” (Microsoft), "Dream Home” (LG), "Digital Home" (Samsung) e "UbiHome" (KJIST) (GHAFFARIANHOSEINI et al., 2013). A casa adaptativa tem como objetivo prever as necessidades do usuário, aprender os padrões de vida e ações do usuário. As características da casa adaptativa abordam um sistema de conforto residencial com conexão entre temperatura, iluminação, ventilação, controles de temperatura de água e padrões do usuário; e um sistema de previsão e controle do ambiente automaticamente. A casa consciente desenvolve serviços para idosos com base na percepção humana. Apresenta como características uma especificação do domínio de casa consciente - sugerindo um domínio específico de aplicação determinando um grupo alvo, como os idosos, e desenvolvendo aplicações apropriadas para o grupo alvo; e um sistema de aprendizado de contexto e detecção - coletando as informações dos residentes usando sensor ou câmera (neste caso os sensores precisam ser escondidos da visão do residente ou pode ser instalado no corpo humano em formas vestíveis, como colar ou relógio). A "House-n Project" visa construir uma casa personalizada que inclua o sistema doméstico com características de adaptabilidade (ambiente personalizado), sistema doméstico personalizado (rede doméstica, aparelhos digitais, novos materiais de construção), ambiente interativo de interface do usuário (onde a interação entre os moradores e sua casa é possível) e projeto arquitetônico de ambiente de visualização (fornecendo interfaces digitais e ambiente de simulação de projeto). A "Easy Living" desenvolve protótipos e tecnologia apropriada para ambientes inteligentes onde a comunicação 
ativa é fornecida entre humano e humano, humano e computador, ou humano e dispositivo. A "Dream Home" proporciona um ambiente como um agente inteligente para os residentes promovendo um ambiente de cozinha em que os moradores possam controlar eletrodomésticos remotamente através da Internet. A "Digital Home” especifica quatro domínios principais para a vida em casa, como viver feliz, divertido, surpreendente e conveniente. Proporcionando aos moradores o ambiente que pode satisfazer a vivência desses quatro temas através de controle de iluminações e temperatura, de fornecimento de um serviço de diagnóstico médico remoto, de fornecimento de menus de jantar para a família automaticamente, do uso de HDTV e sistema de áudio, do uso de dispositivos multimídia, de fornecimento de controle remoto de eletrodomésticos de fora e de fornecimento de GPS. E a "UbiHome" fornece uma infraestrutura para gerenciamento e administração de vários sensores, contexto e aplicação usando computadores distribuídos no ambiente, acompanhando ou notificando a localização, gesto, identidade e intenção do residente, bem como o reconhecimento de um objeto e sua localização. (JANG; LEE, WOO, 2001; JEONG, 2009)

$\mathrm{Na}$ literatura, várias são os itens usados para caracterizar uma smart-home. Observa-se a presença de soluções IOT e de tecnologia de comunicação e informação. Essas características usadas para diversas finalidades de uso da casa e de perfis diferentes de usuários podem se tornar itens de automação residencial na customização em massa de edifícios residenciais.

\section{RESULTADOS E DISCUSSÃO}

A CM na indústria da construção civil, voltada para edifícios residenciais, já apresenta, na literatura e na prática, os itens que são comumente customizados pelos clientes e que se referem aos aspectos físicos e estruturais como áreas externas e layout interno (MARCHESI; MATT, 2017). Diante da evolução da tecnologia de comunicação e informação, é relevante analisar os itens que caracterizam uma smart-home visando como potencial de agregar a customização em edifícios residenciais.

Como visto na revisão teórica esses itens podem ser referenciados como sistemas de equipamentos que possuem funções de suporte, monitoramento, terapia e conforto (CHAN et al., 2008). Podem ser encontrados itens relacionados a serviços como assistência à saúde, eficiência energética, conforto e conveniência, entretenimento, comunicação e segurança (BALTA-OZKAN et al., 2013). E, ainda, relaciona-se os itens com os sistemas inteligentes de uma smart-home como sistema de layout interior, sistema de fachada, sistema de hidráulica e 
drenagem, sistema e gerenciamento de energia, sistema de controle de iluminação, sistema de controle de acesso e monitoramento de segurança, sistema integrado de gerenciamento de dados (GHAFFARIANHOSEINI et al., 2013). Apesar dos estudos apresentarem categorias com nomes diferentes para agrupar os itens que caracterizam a smart-home, as categorias apresentam finalidades semelhantes com itens similares (Quadro 1).

Quadro 1 - Categorias e itens/características de smart-home

\begin{tabular}{|c|c|c|}
\hline Categoria & Itens / características & Autores \\
\hline \multirow{6}{*}{ Conforto } & Eletrodomésticos inteligentes & $\begin{array}{l}\text { CHAN et al., 2008; GHAFFARIANHOSEINI et } \\
\text { al., } 2013\end{array}$ \\
\hline & Objetos inteligentes (ex.: armários) & CHAN et al., 2008 \\
\hline & Equipamentos (ex.: câmera) & $\begin{array}{l}\text { CHAN et al., 2008; GHAFFARIANHOSEINI et } \\
\text { al., } 2013\end{array}$ \\
\hline & $\begin{array}{l}\text { Equipamentos de lazer (TV, cinema em casa, } \\
\text { sistema de áudio, multimídia) }\end{array}$ & CHAN et al., 2008 \\
\hline & $\begin{array}{l}\text { Sistema comunicação interativa (usuários com } \\
\text { pessoas externas e usuário com a casa) }\end{array}$ & BALTA-OZKAN et al., 2013; CHAN et al., 2008 \\
\hline & $\begin{array}{l}\text { Controle (janelas e portas, temperatura, } \\
\text { iluminação, ar condicionado, aquecimento, } \\
\text { temperatura da água, ventilação) }\end{array}$ & $\begin{array}{l}\text { BALTA-OZKAN et al., 2013; CHAN et al., 2008; } \\
\text { GHAFFARIANHOSEINI et al., } 2013\end{array}$ \\
\hline \multirow{2}{*}{ Segurança } & Controle de presença & BALTA-OZKAN et al., 2013 \\
\hline & Controle de janelas e portas e de iluminação & BALTA-OZKAN et al., 2013 \\
\hline \multirow{2}{*}{ Eficiência energética } & Mecanismos automatizados de consumo de energia & BALTA-OZKAN et al., 2013 \\
\hline & $\begin{array}{l}\text { Acesso a dados do tempo de uso de energia e da } \\
\text { conta de energia }\end{array}$ & BALTA-OZKAN et al., 2013 \\
\hline \multirow{4}{*}{$\begin{array}{l}\text { Monitoramento / } \\
\text { assistente de vida / casa } \\
\text { adaptativa }\end{array}$} & Sensores infravermelhos & $\begin{array}{l}\text { CHAN et al., 2008; GHAFFARIANHOSEINI et } \\
\text { al., } 2013\end{array}$ \\
\hline & Sensores vestíveis & $\begin{array}{l}\text { CHAN et al., 2008; GHAFFARIANHOSEINI et } \\
\text { al., } 2013\end{array}$ \\
\hline & Sensores externos (sinais fisiológicas/vitais) & $\begin{array}{l}\text { CHAN et al., 2008; GHAFFARIANHOSEINI et } \\
\text { al., } 2013\end{array}$ \\
\hline & Serviço de diagnóstico médico remoto & GHAFFARIANHOSEINI et al., 2013 \\
\hline \multirow{5}{*}{$\begin{array}{l}\text { Suporte / assistente de } \\
\text { vida / casa adaptativa }\end{array}$} & Interface personalizada & $\begin{array}{l}\text { CHAN et al., 2008; GHAFFARIANHOSEINI et } \\
\text { al., } 2013\end{array}$ \\
\hline & Controle remoto & CHAN et al., 2008 \\
\hline & Robôs de companhia & CHAN et al., 2008 \\
\hline & Tela tátil & CHAN et al., 2008 \\
\hline & Alarme visível & CHAN et al., 2008 \\
\hline \multirow{3}{*}{$\begin{array}{l}\text { Entrega / assistente de } \\
\text { vida / casa adaptativa }\end{array}$} & Entrega de medicamentos em casa automatizada & CHAN et al., 2008 \\
\hline & $\begin{array}{l}\text { Entrega de equipamentos médicos (ex.: } \\
\text { reabilitação) }\end{array}$ & CHAN et al., 2008 \\
\hline & Acionamento de um cuidador & BALTA-OZKAN et al., 2013 \\
\hline Atividade física & Dispositivos fitness & CHAN et al., 2008 \\
\hline \multirow{2}{*}{ Estilos de casa } & Aparelhos digitais & $\begin{array}{l}\text { GHAFFARIANHOSEINI } \text { et al., 2013; JANG; } \\
\text { LEE, WOO, 2001; JEONG, } 2009\end{array}$ \\
\hline & Novos materiais de construção & $\begin{array}{l}\text { GHAFFARIANHOSEINI et al., 2013; JANG; } \\
\text { LEE, WOO, 2001; JEONG, } 2009\end{array}$ \\
\hline
\end{tabular}




\section{CONCLUSÃO}

Este artigo apresentou estudos que caracterizam uma smart-home, bem como estudos que apresentam abordagens e finalidades diferentes sobre smart-home. As características apresentadas na revisão teórica e no quadro de discussão demonstram ser potenciais itens de automação residencial para a customização em massa em edifícios habitacionais, diante da caracterização da smart-home em função do tipo de usuário.

A contribuição teórica deste artigo refere-se à síntese dos itens que caracterizam uma smarthome, bem como análise daqueles itens que são comuns em mais de um estudo. Contribuição prática, deste estudo, refere-se à possibilidade de apresentar mais itens de customização na indústria civil, além daqueles itens físicos e estruturais.

\section{REFERENCIAS}

BALTA-OZKAN, N.; DAVIDSON, R.; BICKET, M.; WHITMARSH, L. Social barriers to the adoption of smart homes. Energy Policy, v. 63, p. 363-374, 2013.

BHATT, J.; VERMA, H. K. Design and development of wired building automation systems. Energy and Buildings, v. 103, p. 396-413, 2015.

CHAN, M.; ESTÈVE, D.; ESCRIBA, C.; CAMPO, E. A review of smart-homes - present states and future challenges. Computer and methods and biomedicine, v. 91, n. 1, p. 55-81, 2008.

DAUM, D.; HALDI, F.; MOREL, N. A personalized measure of thermal comfort for building controls. Building and Environment, v. 46, p. 3-11, 2011.

DAVIS, S. Future Perfect. 1ed. Reading: Addison Wesley P, 1987.

FETTERMANN, D. C.; TORTORELlA, G. L.; TABOADA, C. M. Mass Customization Process in Companies from the Housing Sector in Brazil. In: Cortés-Robles G., García-Alcaraz J., Alor-Hernández G. (eds). Managing Innovation in Highly Restrictive Environments. Cham: Springer, 2019. E-book.

FOGLIATTO, F. S.; SILVEIRA, G. Da; BORENSTEIN, D. The mass customization decade: na update review of the literature. International Journal of Production Economics, n. 1, v. 138, p. 14-25, 2012.

GHAFFARIANHOSEINI, A.; DAHLAN, N. D.; BERARDI, U.; GHAFFARIANHOSEINI, A.; MAKAREMI, N. The essence of future smart houses: from embedding ICT to adapting to sustainability principles. Renewable and Sustainable Energy Reviews, v. 24, p. 593-607, 2013.

JANG, S.; LEE, S.; WOO, W. Research activities on smart environment. DBpia; v. 28, n. 12, p. 85-97, 2001.

JEONG, K. Design and operation of smart homes in USA and Korea, US: Purdue University, 2009.

KIM, Y.; LEE, J.; LEE, H. Life-log data-based window opening and closing for individual customized services in symbiosis houses. Procedia Environmental Sciences, v. 22, p. 247-256, 2014.

KOTHA, S. Mass customization: implementing the emerging paradigm for competitive advantage. Strategic Management Journal. v. 16, Summer, p. 21-42, 1995. 
LEE, J. H.; LEE, H.; KIM, M. J.; WANG, X.; LOVE, P. E. D. Context-aware inference in ubiquitous residential environments. Computers in Industry, v. 65, n. 1, p. 148-157, 2014.

PARK, C. K.; KIMC, H. J.; KIM, Y. S. A study of factors enhancing smart grid consumer engagement. Energy Policy, v. 72, p.211-218, 2014.

PARK, M.; YOO, J. Benefits of mass customized products: moderating role of product involvement and fashion innovativeness. Heliyon, n. 2, v. 4, p. 1-25, 2018.

PILlER, F. T.; LINDGENS, E.; STEINER, F. Mass Customization at Adidas: Three Strategic Capabilities to Implement Mass Customization. Working Papers Series, January 29, 2012.

PINE II, B. J. Mass Customization: the new frontier in business competition. Boston: Harvard Business School Press, 1993.

POLLARD, D.; CHUO, S.; LEE, B. Strategies for Mass Customization. Journal of Business \& Economics Research, v.6, n.7, p.77-86, July 2008.

SCHOENWITZ, M.; POTTER, A.; GOSLING, J.; NAIM, M. Product, process and customer preference alignment in prefabricated house building. International Journal of Production Economics, v. 183, part. A, p. 79-90, 2017.

SCHOENWITZ, M.; NAIM, M.; POTTER, A. The nature of choice in mass customized house building. Construction Management and Economics, n. 3, v. 30, p. 203-219, 2012.

SHAH, J.; MISHRA, B. Customized IoT enabled Wireless Sensing and Monitoring Platform for Smart Buildings. Procedia Technology, v. 23, p. 256 - 263, 2016.

SILVEIRA, G. Da; BORENSTEIN, D.; FOGLIATTO, F. S. Mass customization: Literature review and research directions. International Journal of Production Economics, n. 1, v. 72, p. 1-13, 2001.

TEICHMANN, K.; SCHOLL-GRISSEMANN, U.; STOKBURGER-SAUER, N. E. The Power of Codesign to Bond Customers to Products and Companies: The Role of Toolkit Support and Creativity. Journal of Interactive Marketing, v. 36, p. 15-30, 2016.

WAN, X.; WANG, T.; ZHANG, W.; CAO, J. Perceived value of online customization experience in China: Concept, measurement, and consequences. The Journal of High Technology Management Research, v. 28, n.1, p. 17-28, 2017.

WANG, Y.; MA, H. S.; YANG, J. H.; WANG, K. S. Industry 4.0: a way from mass customization to mass personalization production. Advances in Manufacturing, v. 5, n. 4, p. 311-320, 2017.

WANG, Z.; ZHANG, M.; SUN, H.; ZHU, G. Effects of standardization and innovation on mass customization: an empirical investigation. Technovation, v. 48, p. 79-86, 2016.

YOO, J.; PARK, M. The effects of e-mass customization on consumer perceived value, satisfaction, and loyalty toward luxury brands. Journal of Business Research, n. 12, v. 69, p. 5775-5784, 2016.

ZIPKIN, P. H. The limits of mass customization. MIT Sloan Management Review, v.42, n.3, p.81-87, Spring 2001. 